\title{
Estimation of laser weld parameters using surrogate modelling technique
}

\author{
Karel Pavlíček, Václav Kotlan, Ivo Doležel *
}

\begin{abstract}
A surrogate technique based on Gaussian Process (GP) is used for predicting quality of laser in case of laser welding process that may be supported by induction preheating. FEM-based solution of the problem is computationally expensive because it combines computation of 3D coupled nonlinear electromagnetic and temperature fields. The quality of laser welds is quantified with weld depth, which depends on a number of input parameters. The paper deals with two of them - thickness of the welded steel sheet and power of the laser beam. First, selected FEM simulations allow finding data describing the dependency between the two input parameters and weld depth. These data allow creating a surrogate model that is able to predict weld depth at any point close to the points where the results are known. The principal goal is to essentially save the computational time. The surrogate model also allows estimating prediction plausibility and running the full FEM calculation in cases where the prediction is not sufficiently accurate. The methodology is illustrated with a typical example whose results are discussed. field

K e y w ords: laser welding, surrogate model, approximation, numerical analysis, temperature field, electromagnetic
\end{abstract}

\section{Introduction}

Various surrogate modelling techniques are used for numerical solution of physical problems in the domain of electrical engineering, especially in applications where fast obtaining of sufficiently accurate results is required. A typical application is laser welding of two steel sheets. One of the most important parameters of the weld is its depth. This depth depends on many input parameters of the laser welding process. In this paper, the principal attention is paid to the influence of thickness of the welded sheet and power of the laser beam.

The depth of the weld can provide information about the weld quality. Its determination is usually carried out by the computer simulation, using, eg the finite element method (FEM). But in most cases, the geometrical arrangement is complex and the task must be calculated in three dimensions. Such computations are, of course, expensive, as they can take a number of hours or even days. In industrial applications, for instance, we work with many kinds of steel sheets of various dimensions. And receiving results of FEM simulation for all existing variants would be extremely time demanding.

The idea presented in this paper is to calculate the FEM-based results just for several important variants characterized by the smallest and biggest thickness of sheets (and also few values between them), together with selected values of laser power. The results are then used for predicting results in all other arrangements. This goal is reached by applying an appropriate surrogate modelling technique which also provides a probability that these results are in a prescribed interval of tolerance.

\section{Theory of surrogate models}

\subsection{Surrogate modelling - general considerations}

Surrogate modelling has a wide spectrum of applications in many technical domains. It is one of the alternatives to conventional modelling techniques used when classical modelling methods are computationally too expensive or when they are not suitable from different reasons. Those methods are based on the idea of replacing a complex model with another one based on previous knowledge of expected results or measured data, fitting of measured data to create model has been recently studied, $e g$ in [1]. Nice definition the of surrogate model is written in [2]: "A surrogate model is a mathematical model that mimics the behavior of a computationally expensive simulation code over the complete parameter space as accurately as possible, using the least amount of data points."

The relation of the surrogate model and "complete" model can be described by expression [3]:

$$
f^{\prime}(\mathbf{x})=f(\mathbf{x})+e(\mathbf{x}),
$$

where $f^{\prime}(\mathbf{x})$ is the surrogate model, $f(\mathbf{x})$ is the source (complete) model and $e(\mathbf{x})$ is the error of approximation.

In case that the model $f(\boldsymbol{x})$ is not intended to be known and only inputs and outputs are taken into consideration, the solved task is called black box modelling [3]. The alternative to surrogate modelling in area of less computationally expensive models is Model Order Reduction. The difference between surrogate modelling and model order reduction consists in the fact that the model

\footnotetext{
* Department of Theory of Electrical Engineering, University of West Bohemia in Pilsen, Pilsen, Czech Republic, \{pavlk, vkotlan, idolezel\}@kte.zcu.cz
} 
order reduction performs reduction of the input parameters of the model and surrogate modelling is meant to be as accurate as possible [4].

Various methods can be used to create a surrogate model. Very common techniques are, for example, polynomial approximation, kriging, radial basis functions method or support vector regression $[2,3]$. Each of the above techniques exhibits various advantages and disadvantages. Comparison of these techniques has been made in $[2]$.

The method called kriging (Gaussian Process) is used for surrogate model creation in the paper. Therefore, it is briefly described to provide a short introduction to it.

\subsection{Regression using Gaussian process (Kriging)}

Gaussian processes (GP) belong to the trends which are used in the domain of supervised machine learning for regression and classification tasks. For creation of a surrogate model it is relevant the regression task formulation that predicts dependency of continuous output variable on the input data of vector (or scalar) quantity.

Input and output variables are grouped in datasets, which can be defined as follows: $D=\left\{\mathbf{x}=\left(\mathbf{x}_{1}, \ldots \mathbf{x}_{n}\right)^{\top}\right.$, $\left.\boldsymbol{y}=\left(y_{1}, \ldots y_{m}\right)^{\top}\right\}$

For each regression analysis (surrogate model creation) there usually exist two datasets. The first one is used to train the data and the second is used to make validation of the predicted output variable. Existence of the training data puts the method to the group of supervised machine learning algorithms. Surrogate modelling uses simulation results from the high fidelity model as training and validation dataset.

The Gaussian process is based on the probabilistic principle and, therefore, it also allows obtaining information about probability of the predicted output variable. Each Gaussian process is defined by two functions. The first function is called mean function $m(\mathbf{x})$ and second function is called covariance function $k\left(\mathbf{x}, \mathbf{x}^{\prime}\right)$. Another name often used for covariance function is kernel function. That is why it is also classified as one of the kernel methods.

$$
f(\mathbf{x}) \sim \operatorname{GP}\left(m(\mathbf{x}), k\left(\mathbf{x}, \mathbf{x}^{\prime}\right)\right) .
$$

In regression tasks it is often taken into account $m(\mathbf{x}=0)[5,6]$.

As a kernel, one of various functions can be used. Very common it is the squared exponential kernel [7]:

$$
k\left(\mathbf{x}, \mathbf{x}^{\prime}\right)=\sigma_{\mathrm{f}}^{2} \exp \left[\frac{-\left|\mathbf{x}-\mathbf{x}^{\prime}\right|^{2}}{2 l^{2}}\right] .
$$

From the formula it is obvious that the output of the covariance function depends on the input data and so called hyperparameters, which are, in the case of squared exponential kernel, the values $\sigma_{\mathrm{f}}$ and $l$.

One of the tasks is to find the correct value of hyperparameters. This is possible, eg using the maximum likelihood estimation from the training dataset. At first, it is necessary to select their initial values which are then iteratively optimized (eg using the conjugate gradient method). To avoid issue with local minima, it is possible to perform optimization with several different starting data [5].

When the covariance function is applied to the input dataset, the covariance matrix is created in the form

$$
\mathbf{K}=\left(\begin{array}{cccc}
k\left(\mathbf{x}_{1}, \mathbf{x}_{1}\right) & k\left(\mathbf{x}_{1}, \mathbf{x}_{2}\right) & \ldots & k\left(\mathbf{x}_{1}, \mathbf{x}_{n}\right) \\
k\left(\mathbf{x}_{2}, \mathbf{x}_{1}\right) & k\left(\mathbf{x}_{2}, \mathbf{x}_{2}\right) & \ldots & k\left(\mathbf{x}_{2}, \mathbf{x}_{n}\right) \\
\vdots & \vdots & \vdots & \vdots \\
k\left(\mathbf{x}_{n}, \mathbf{x}_{1}\right) & k\left(\mathbf{x}_{n}, \mathbf{x}_{2}\right) & \ldots & k\left(\mathbf{x}_{n}, \mathbf{x}_{n}\right)
\end{array}\right)
$$

This covariance matrix is then used together with vector $\boldsymbol{k}^{*}$ for actual prediction of the output variable at the input point $\boldsymbol{x}_{*}$.

$$
\boldsymbol{k}^{*}\left(\boldsymbol{x}_{*}\right)=\left(k\left(\boldsymbol{x}_{*}, \mathbf{x}_{1}\right), \ldots, k\left(\mathbf{x}_{*}, \mathbf{x}_{n}\right)\right)^{\top}
$$

Prediction of output variable $\hat{y}$ at point $\boldsymbol{x}_{*}$ is then

$$
\begin{gathered}
\hat{y}\left(\boldsymbol{x}_{*}\right)=\boldsymbol{k}^{* \top}\left(\mathbf{x}_{*}\right) \mathbf{K}^{-1} \boldsymbol{y} \\
\sigma_{\hat{y}}^{2}\left(\mathbf{x}_{*}\right)=k\left(\mathbf{x}_{*}, \mathbf{x}_{*}\right)-\boldsymbol{k}^{* \top}\left(\mathbf{x}_{*}\right) \mathbf{K}^{-1} \boldsymbol{k}^{*}\left(\mathbf{x}_{*}\right) .
\end{gathered}
$$

The above text describes one of many formulations of the Gaussian processes for regression tasks [7] that was selected because its implementation in software [8] is advantageous. Another interesting alternative formulation has been made in [9].

\section{Laser welding}

\subsection{Laser weld parameters}

The main advantages of laser welding are narrow seam with high weld depth and minimal residual mechanical stress of thermal origin in the material [10]. Due to these advantages, laser welding in the industry is used very widely and only one key question, weldability of materials, has to be satisfied.

The weld/seam quality is influenced by many factors. For example the following parameters affect the quality of the weld/seam [10]:

- laser power,

- welding speed,

- position of the focus,

- inert gas (if used),

- material thickness,

- surface properties.

Many studies were performed to evaluate the welding process and the quality of the weld itself. For example, study about this topic in the area of electrical engineering has been carried out in [11] where the evaluated process of welding laminated electrical steel packets is used in electrical machines. 


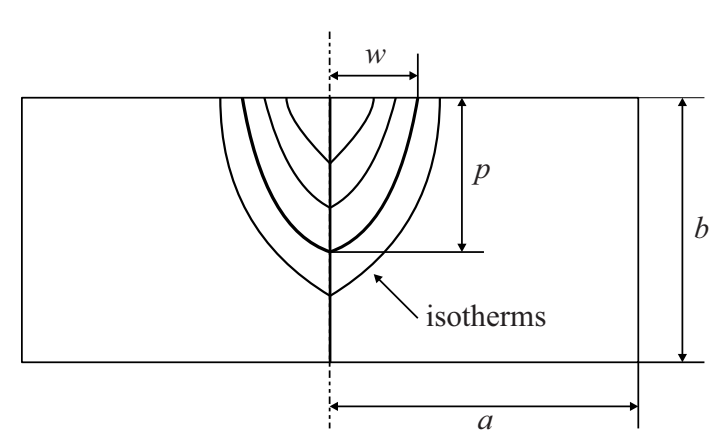

Fig. 1. Temperature distribution in the area of weld

The task can be also to improve the weld depth, which can be achieved using predictive control of laser welding process [10]. Reference [12] investigates parameters affecting the welding process and the weld depth: intensity, interaction time and specific power. To quantify the process, another parameter called power factor is used.

The most investigated parameter of the weld is called weld depth. To measure weld depth physically, material must be destroyed [10]. That is why computer modelling of the phenomena is very important because it allows determining weld depth without performing any complicated measurement.

\subsection{Laser weld temperature distribution}

Figure 1 presents the temperature distribution in the cross section of the weld. Particular lines are isotherms providing information about spots with the same temperature. The most important isotherm (for steel S355 that is the subject of our research) has the value of $780^{\circ} \mathrm{C}$ because this isotherm is used for determining the weld parameters. Parameter $w$ is the width of the weld and $p$ is its depth that is studied in this paper.

After the laser welding process, three different results can occur: good quality laser weld Fig. 2(a), weld penetrates completely the material Fig. 2(b), two sheets are welded together but laser weld has bad mechanical properties Fig. 2(c).

\section{Mathematical model}

The process of combined laser welding represents a coupled, strongly non-linear problem characterized by an interaction of the magnetic and temperature fields mutually influencing one another

\subsection{Magnetic field produced by inductor}

Magnetic field produced by the heater may be described in terms of several different quantities. We used the formulation based on the magnetic vector potential $\boldsymbol{A}$ in the form

$$
\operatorname{curl}\left(\frac{1}{\mu} \operatorname{curl} \mathbf{A}\right)+\gamma\left(\frac{\partial \mathbf{A}}{\partial t}-\mathbf{v} \times \operatorname{curl} \mathbf{A}\right)=\mathbf{J}_{\text {ext }},
$$

where $\mu$ is the permeability that depends (in ferromagnetic parts) on applied magnetic field, $\gamma$ stands for the electric conductivity, $\mathbf{v}$ denoted the velocity of the substrate and $J_{\text {ext }}$ is the vector of the external current density in the inductor. Both parameters $\mu$ and $\gamma$ are temperature-dependent functions and these functions are generally nonlinear.

But solution to (1) in 3D is still practically unfeasible. The principal reason consists in a deep disproportion between the frequency $f$ (usually on the order of $10^{2}-10^{4} \mathrm{~Hz}$ ) of the field current $I_{\text {ext }}$ carried by the inductor and time of preheating or postheating (which usually takes several seconds). The computation of one variant respecting all relevant nonlinearities would take, therefore, unacceptable time on the order of days. That is why the model had to be somewhat simplified, without any substantial influence on the results.

First, the magnetic field is supposed to be harmonic, which allows solving the problem in the frequency domain. The magnetic permeability of ferromagnetic parts is, however, not supposed to be a constant everywhere; its value is always assigned to the local value of magnetic flux density $B$ in every element of the discretization mesh. Then the magnetic field distribution can be described in terms of the phasor $\underline{\boldsymbol{A}}$ and the corresponding equation reads

$$
\operatorname{curl}(\operatorname{curl} \underline{\boldsymbol{A}})+\gamma \mu(j \omega \underline{\boldsymbol{A}}-\mathbf{v} \times \operatorname{curl} \underline{\boldsymbol{A}})=\mu \underline{\boldsymbol{J}}_{\mathrm{ext}},
$$

where $\omega$ represents the angular frequency $(\omega=2 \pi f)$.

Second, the velocity $v$ of the plate does not exceed several millimeters or a few centimeters per second. That is why the the last term of the left-hand side of (9) can be neglected without any substantial error. Then, the basic equation to be solved has the form

$$
\operatorname{curl}(\operatorname{curl} \underline{\boldsymbol{A}})+j \gamma \mu \omega \underline{\boldsymbol{A}}=\mu \underline{\boldsymbol{J}}_{\mathrm{ext}} .
$$

The boundary condition along a sufficiently distant boundary is of the Dirichlet type $(\underline{\boldsymbol{A}} \times \boldsymbol{n}=\boldsymbol{0}$, where $\boldsymbol{n}$ denotes the vector of normal to the surface). In case of any space symmetry, the corresponding interface is described by the Neumann condition.
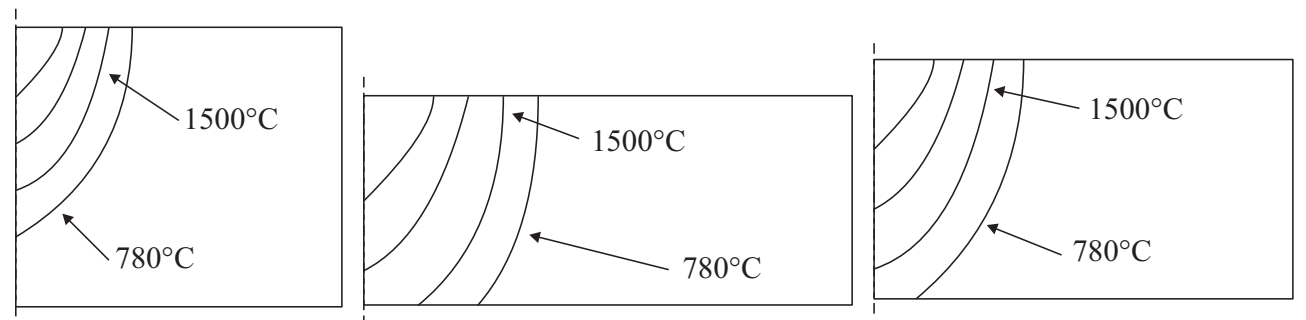

Fig. 2. Temperature distribution in the area of weld 


\subsection{Temperature field generated by heater}

The temperature field generate by the heating inductor is described by the heat transfer equation, taking into account the influence of motion. It may be written in the form

$$
\operatorname{div}(\lambda \operatorname{grad} T)=\rho c_{\mathrm{p}}\left(\frac{\partial T}{\partial t}+\mathbf{v} \cdot \operatorname{grad} T\right)-w,
$$

where $\lambda$ denotes the thermal conductivity, $\rho$ stands for the mass density, $c_{\mathrm{p}}$ is the specific heat at constant pressure, $w$ represents the volumetric internal sources of heat in the processed material and $t$ is time. The volumetric heat sources $w$ generally consist of the volumetric Joule losses $w_{\mathrm{J}}$ and specific hysteresis losses $w_{\mathrm{h}}$

$$
\begin{gathered}
w=w_{\mathrm{J}}+w_{\mathrm{h}}, \\
w_{\mathrm{J}}=\frac{\left|\underline{\boldsymbol{J}}_{\text {ind }}\right|^{2}}{\gamma}, \underline{\boldsymbol{J}}_{\text {ind }}=j \omega \gamma \underline{\boldsymbol{A}} .
\end{gathered}
$$

The losses $w_{\mathrm{h}}$ can be determined either from the measured loss dependence for the material used, or from a suitable (for example Steinmetz) analytical formula. But in many cases they can be neglected as their value is just a small fraction of the losses $w_{\mathrm{J}}$.

The boundary condition takes into account both convection and radiation and may be expressed in the form

$$
-\frac{\partial T}{\partial n}=\alpha\left(T_{\mathrm{s}}-T_{0}\right)+\sigma C\left(T_{\mathrm{s}}^{4}-T_{\mathrm{r}}^{4}\right) .
$$

Here, $T_{\mathrm{S}}$ is the temperature of surface of the plate, $\alpha$ denotes the coefficient of the convective heat transfer, $T_{0}$ stands for the temperature of the surrounding medium (air), $\sigma=5.67 \times 10^{-8} \mathrm{Wm}^{-2} \mathrm{~K}^{-4}$ is the Stefan-Boltzmann constant, $C$ represents the emissivity of the heated part and $T_{\mathrm{r}}$ is the temperature of the surface to which heat is radiated. Unlike the magnetic field, the velocity term in (11) cannot be neglected even for very low values.

\subsection{Temperature field produced by laser beam}

Heating by the laser beam is considered in the form of delivery a specified heat power to a given spot of the plate. The basic equation corresponds to (11), but now the internal volumetric losses $w$ vanish. Heat is delivered to material from the part of the surface heated by the laser beam. This may be taken into account by a boundary condition that is similar to (13), but it contains one more term $q_{\text {in }}$ corresponding to the delivered thermal flux

$$
-\frac{\partial T}{\partial n}=-q_{\mathrm{in}}+\alpha\left(T_{\mathrm{s}}-T_{0}\right)+\sigma C\left(T_{\mathrm{s}}^{4}-T_{\mathrm{r}}^{4}\right) .
$$

Prescription of the boundary condition requires knowledge of the actual surface temperature of the body after preheating, which is somewhat reduced by cooling caused by the local time delay between the preheating and laser heating.
As it is often complicated to determine accurately the parameters influencing the coefficient $C$ of emissivity such as multiple reflections or configuration factors, radiation may be respected by a generalized coefficient of convection $\alpha_{\text {gen }}$ in the form

$$
\alpha\left(T_{\mathrm{s}}-T_{0}\right)+\sigma C\left(T_{\mathrm{s}}^{4}-T_{\mathrm{r}}^{4}\right)=\alpha_{\text {gen }}\left(T_{\mathrm{s}}-T_{0}\right) .
$$

The coefficient $\alpha_{\text {gen }}$ must be determined experimentally, using suitable calibration measurements.

\section{Surrogate model}

\subsection{Input data}

Input data for definition of the surrogate model are results obtained using FEM simulation in COMSOL Multiphysics. The results from FEM simulation are used as input dataset, for details regarding the used FEM model, see [13]. The model deals with welding of two sheets made of steel S355.

This process is solved as a transient hard coupled problem (heat and electromagnetic field - due to induction preheating of both plates) with non-linear material properties. Due to geometric arrangement, it must be also solved in three dimensions. Combination of those factors makes the simulation very expensive. The only factor that reduces the computational time is longitudinal symmetry.

From the simulation results, selected data were extracted from the cross-section of the laser weld based on the isotherm, like those depicted in Fig. 1.

Two configurations were solved. The first one contained hybrid laser and induction heating, while the second one just with laser heating.

\subsection{Surrogate model implementation}

Fitting the input data for obtaining surrogate model can be done, apart from implementing the algorithm, in many various scientific tools and systems. Such a system could be, eg MATLAB or one of many open source codes. The authors used Scipy package scikit-learn [8] (in version 0.17), which is an advanced toolkit for machine learning based on the Python programming language environment. It allows using multiple types of algorithms for regression tasks. For the needs of the article, the Gaussian Process regression is used which is, in case of scikit-learn, implemented in the form of class GaussianProcess.

The task then consists of two steps. The first step is fitting the data and the second one is making the prediction.

The function call to perform fit of the input data can be, eg as follows:

from sklearn . gaussian_process import GaussianProcess

import INPUT_DATA

$\mathrm{x}=$ INPUT_DATA. $\mathrm{x}$

$\mathrm{y}=$ INPUT_DATA.y

gp_p $=$ GaussianProcess $($ theta0 $=0.1$,

thetaL $=0.001$

thetaU $=1 .$, ,

nugget $=0.001$ )

gp_p . fit $(x, y)$ 


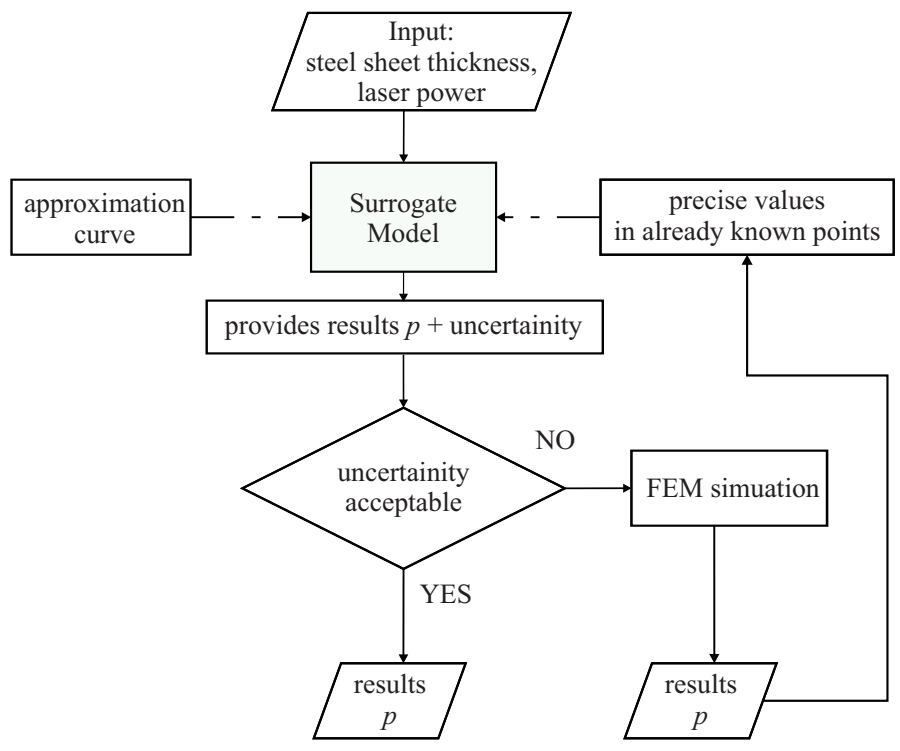

Fig. 3. Proposed algorithm for problem solution

Parameters theta0, thetaL and thetaU are called hyperparamaters and can be used for configuration of the fit. Apart from these parameters, also the following parameters are important:

- regr - sets the regression trend, simple constant trend being default,

- corr - sets the used kernel, squared-exponential autocorrelation model being default,

- beta0 - sets how regression weights are determined, Maximum Likelihood Estimation being default.

When the surrogate model is ready (the process of fitting is finished), then the method predict can be used. Very valuable option of method predict is MSE (Mean Square Error) which, when activated, allows also receiving information about the error of the prediction.

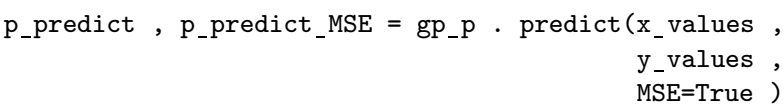

Those functions can be then used for solution of the defined problem. In the case of the problem solved in this paper, the overview of the proposed solution is depicted in the flowchart in Fig 3.

Available input data (from the FEM simulation) are fitted using Gaussian process, so the surrogate model is created. The dependent variable $\mathbf{x}=\left[\mathbf{x}_{1}, \ldots, \mathbf{x}_{n}\right]^{\top}$ and has form of $\mathbf{x}_{i}=\left(b_{i}, P_{i}\right), b$ is the material thickness and $P$ is the power of laser beam.

Using this surrogate model it is possible to predict values of $p$ for values of steel sheet thickness and laser power for such cases, where values obtained by FEM are not available. If the value of MSE is unacceptably high, it is possible to run in this case new FEM simulation to obtain improved results that would improve the surrogate model.

Table 1 presents the points, at which the FEM simulation was carried out and which are used for creation of the surrogate model. The sign || shows how the data are gradually added in the process of learning of the model.
Table 1. Input points for surrogate model creation

\begin{tabular}{c|ccc||cc||cccc}
\hline$b(\mathrm{~mm})$ & 16 & 11 & 6 & 14 & 11 & 18 & 8 & 10 & 14 \\
\hline$P(\mathrm{~W})$ & 325 & 375 & 425 & 425 & 475 & 500 & 525 & 575 & 600 \\
\hline
\end{tabular}

Table 2. Comparison of predicted and FEM simulation results without induction preheating

\begin{tabular}{c|ccccc}
\hline$b(\mathrm{~mm})$ & 15 & 15 & 5.5 & 4.0 & 11 \\
\hline$P(\mathrm{~W})$ & 550 & 400 & 350 & 575 & 500 \\
\hline predicted $p(\mathrm{~mm})$ & 3.579 & 2.935 & 2.795 & 3.562 & 3.397 \\
\hline MSE of $p(\mathrm{~mm})$ & 0.00023 & 0.00020 & 0.00188 & 0.00396 & 0.00018 \\
\hline$p_{\text {FEM }}(\mathrm{mm})$ & 3.561 & 2.959 & 2.732 & 3.562 & 3.383 \\
\hline relative error $(\%)$ & 0.52 & 0.81 & 2.32 & 10.94 & 0.42 \\
\hline
\end{tabular}

\section{Results}

As mentioned before, the surrogate model improves itself with increasing number of the input data. Figure 4 shows the first fit of the input data (obtained from 3 available points - first three points in Table 1). It can be seen that the uncertainty in some areas of the figure is unacceptably high.

After adding two more points (now 5 input points are available) the results are depicted in Fig. 5.

The results for all 9 available input points in Table 1 are in Figs. 6 and 7.

Next task is to check whether the prediction is in a good accordance with the results obtained using highfidelity model (FEM simulation). To do so, several points were selected for comparison. At these points, also the relative errors was calculated (the reference values being taken from FEM simulation results).

At first, values at points for the variant without induction preheating were compared, related to Fig. 6. The results are summarized in Table 2. 


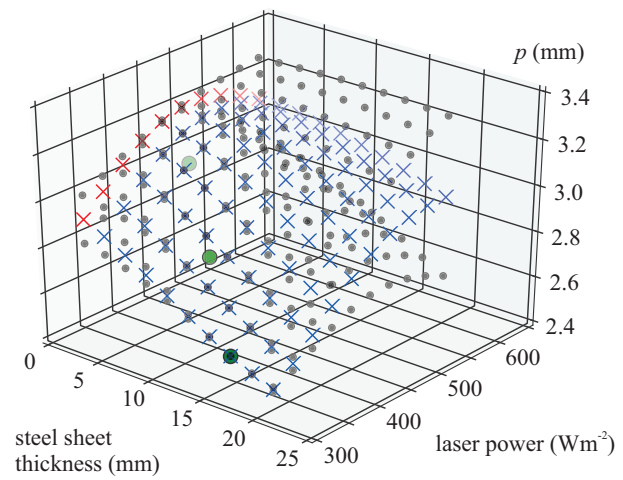

Fig. 4. Results of the prediction (without induction preheating) 3 input points

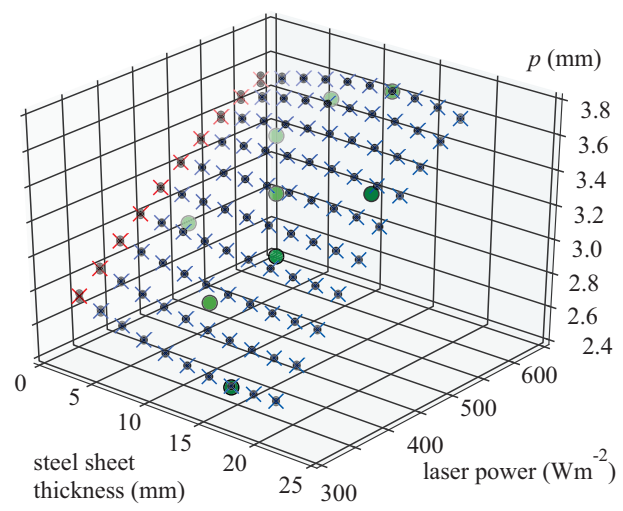

Fig. 6. Results of the prediction (without induction preheating) 9 input points

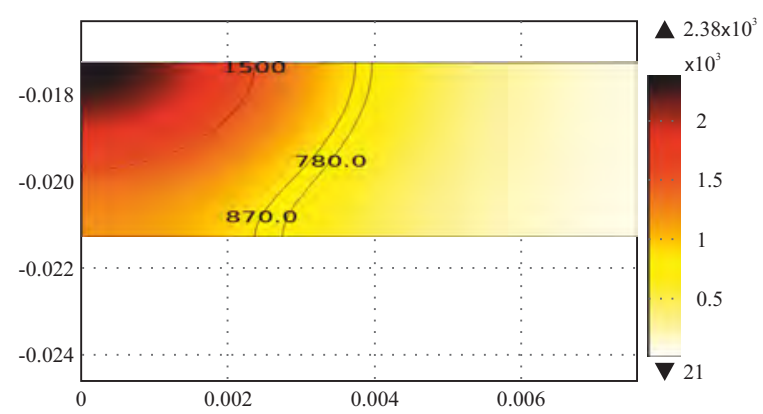

Fig. 8. Temperature distribution in the area of weld $-4 \mathrm{~mm}$ thickness, without induction preheating

Very interesting is the point $(4.0,575)$, where the value of $p_{\text {FEM }}$ is computed as 4.0 , which is exactly equal to the thickness of the plate. Thus, the isotherm $780{ }^{\circ} \mathrm{C}$ touches the bottom surface of the steel sheet. This would mean a weld of low quality, as depicted in Fig. 2(c).

Incorrect variants of the welding process are marked with red crosses in Figs. 4-7. These variants indicate that laser penetrates completely the steel sheet. For the variant with $w=2 \mathrm{~mm}$ and $P=575 \mathrm{~W}$, the temperature distribution is depicted in Fig. 9.

The difference between Figs. 8 and 9 is in visibility of low-quality part of the weld. While Fig. 8 shows the weld with thermally influenced zone through the whole thickness of material that will be brittle and of low quality, it will not be visible because the plate is not welded

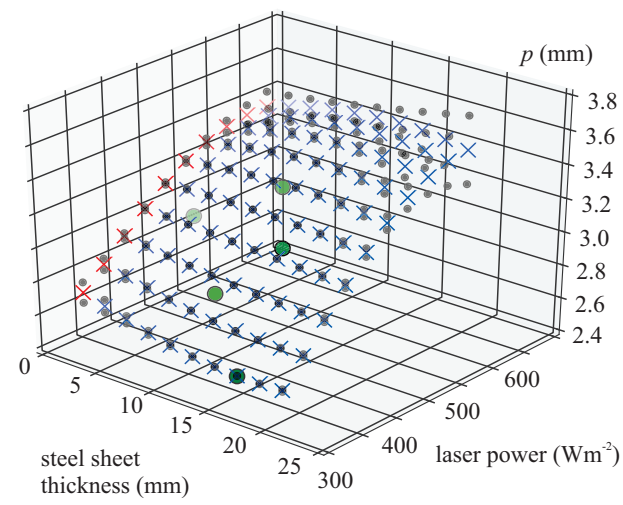

Fig. 5. Results of the prediction (without induction preheating) 5 input points

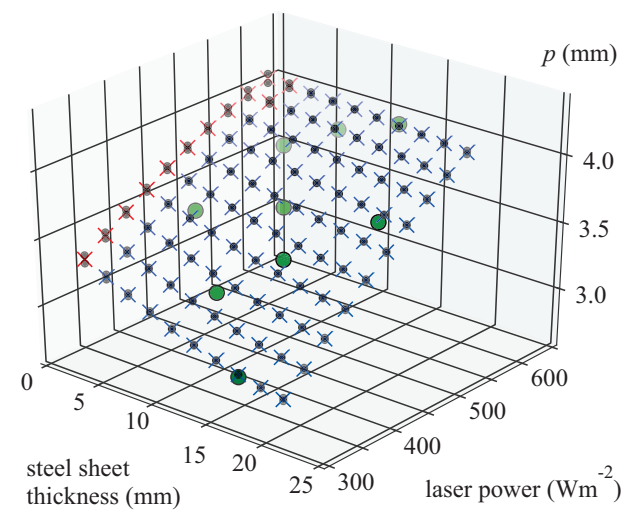

Fig. 7. Results of the prediction (with induction preheating) - 9 input points

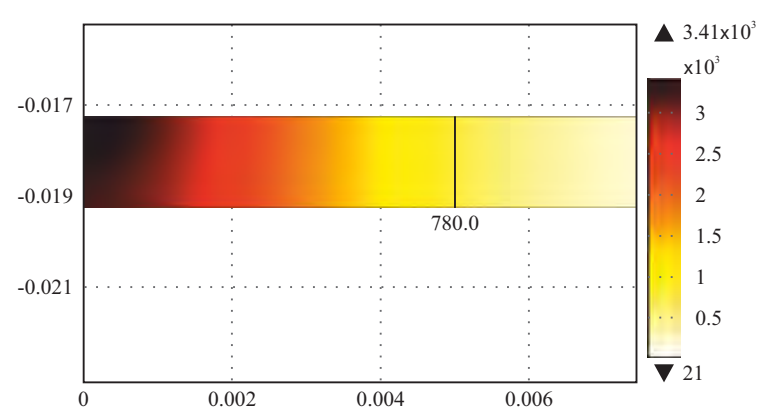

Fig. 9. Temperature distribution in the area of weld $-2 \mathrm{~mm}$ thickness, without induction preheating

through. On the other hand, in case of Fig. 9 the plate will be welded through and material will be completely molten. This difference may be demonstrated even on the metamodel: while for a thin plate the prediction marked by red color is evidently bad, in case of the boundary case (Fig. 8) the prediction is OK, as the weld depth is $3.5 \mathrm{~mm}$ while the thickness is $4 \mathrm{~mm}$. This discrepancy is caused by insufficient information in the metamodel for such a low-quality case.

Validation was also made for variant with the induction preheating. The validation dataset used for it is given in Table 3.

The computations show that from the viewpoint of modelling, it is not much important whether we solve the variant with or without induction preheating. But in 
case of induction preheating, the savings in time are much higher.

Table 3. Comparison of predicted and FEM simulation results with induction preheating

\begin{tabular}{c|ccc}
\hline$b(\mathrm{~mm})$ & 15 & 15 & 11 \\
$P(\mathrm{~W})$ & 550 & 400 & 500 \\
predicted $p(\mathrm{~mm})$ & 3.882 & 3.268 & 3.758 \\
MSE of $p(\mathrm{~mm})$ & 0.00059 & 0.00051 & 0.00048 \\
$p_{\text {FEM }}(\mathrm{mm})$ & 3.827 & 3.233 & 3.720 \\
relative error $(\%)$ & 1.45 & 1.09 & 1.03 \\
\hline
\end{tabular}

\section{Conclusion}

The proposed solution based on metamodelling/surrogate modelling provides promising results. It is clear that in principle it can not replace solution of "full" FEM model, but obtaining results very fast makes such an approach interesting. One of the advantages of the proposed solution is that it can provide information about probability of the prediction and it can run eventually the calculation if prediction is not "plausible" enough. The methodology is quite versatile and fast. But in case of problems leading to large covariance matrices, the Gaussian process loses its most substantial advantage - speed. Nevertheless all the computations and predictions are not trustworthy until they are verified (validated) using the experimental results. This is next aim of the work, to obtain measurement data and compare it with the calculated values.

\section{Acknowledgment}

This research has been supported by the Ministry of Education, Youth and Sports of the Czech Republic under the RICE New Technologies and Concepts for Smart Industrial Systems, project LO1607.

\section{REFERENCES}

[1] E. Pastuchová and M. Zákopčan, "Comparison of Algorithms for Fitting a Gaussian Function used in Testing Smart Sensors", Journal of Electrical Engineering, vol. 66, no. 3, pp. 178-181, 2015.

[2] A. Diaz-Manriquez, G. Toscano-Pulido, and W. Gomez-Flores, "On the Selection of Surrogate Models in Evolutionary Optimization Algorithms", In 2011 IEEE Congress of Evolutionary Computation (CEC), pp. 2155-2162, June 2011.

[3] G. Montemayor-Garca and G. Toscano-Pulido, "A Study of Surrogate Models for their Use in Multiobjective Evolutionary Algorithms", In 2011 8th International Conference on Electrical Engineering, Computing Science and Automatic Control, pp. 1-6, Oct 2011.

[4] M. B. Yelten, T. Zhu, S. Koziel, P. D. Franzon, and M. B. Steer, "Demystifying Surrogate Modelling for Circuits and Systems", IEEE Circuits and Systems Magazine, vol. 12, no. 1, pp. :45-63, Firstquarter 2012.
[5] C. K. I. Williams and C. E. Rasmussen, "Gaussian Processes for Regression", In Advances in neural information processing systems, pp. 514-520, 1996.

[6] C. M. Bishop, "Pattern Recognition and Machine Learning (Information Science and Statistics)", Springer-Verlag New York, Inc., Secaucus, NJ, USA, 2006.

[7] C. E. Rasmussen and C. K. I. Williams, "Gaussian Processes for Machine Learning (Adaptive Computation and Machine Learning series)", The MIT Press, 2005.

[8] F. Pedregosa, G. Varoquaux, A. Gramfort, V. Michel, B. Thirion, O. Grisel, M. Blondel, P. Prettenhofer, R. Weiss, V. Dubourg, J. Vanderplas, A. Passos, D. Cournapeau, M. Brucher, M. Perrot and E. Duchesnay, "Scikit-learn: Machine Learning in Python", Journal of Machine Learning Research, vol. 12 pp. 2825-2830, 2011.

[9] M. H. S. Mendes, G. L. Soares, J. L. Coulomb, and J. A. Vasconcelos, "Appraisal of Surrogate Modelling Techniques: A Case Study of Electromagnetic Device", IEEE Transactions on Magnetics, vol. 49, no. 5, pp. 1993-1996, May 2013.

10] A. Bollig, D. Abel, C. Kratzsch, and S. Kaierle, "Identification and Predictive Control of Laser Beam Welding using Neural Networks", In 2003 European Control Conference (ECC), pp. 2457-2462, Sep 2003.

11] T. Schade, R. M. Ramsayer, and J. P. Bergmann, "Laser Welding of Electrical Steel Stacks Investigation of the Weldability" In 2014 4th International Electric Drives Production Conference (EDPC), pp. 16, Sep 2014.

12] S. Williams and W. Suder, "Use of Fundamental Laser Material Interaction Parameters in Laser Welding", In CLEO: 2011-Laser Science to Photonic Applications, pp. 1-2, May 2011.

13] V. Kotlan, R. Hamar, D. Panek and I. Doležel, "Combined Heat Treatment of Metal Materials", COMPEL: The International Journal for Computation and Mathematics in Electrical and Electronic Engineering, vol. 35 no. 4, pp. 1450-1459, 2016.

Received 18 January 2018

Karel Pavlíček born in Pilsen, Czech Republic, recieved BEng and MSc in Electrical Engineering from the University of West Bohemia, in 2011 and 2013 respectively. He is currently a PhD student at the University of West Bohemia, Pilsen. His professional and research interests are numerical simulations in the area of electrical engineering and its applications to the real world problems and challenges.

Václav Kotlan born in 1979 in Klatovy, Czech Republic, graduated from the Faculty of Electrical Engineering (University of West Bohemia Pilsen, Czech Republic) in 2003. In 2008 he received his PhD degree in Power Engineering, from 2015 is Associate Professor. Research interests are electrical power systems (transmission line parameters, numerical methods, transients on transmission lines) and also numerical modelling of electromagnetic and coupled fields. He published more than 60 papers in scientific journals and conference proceedings.

Ivo Doležel was born in 1949. He received the Ing degree in electrical engineering from Czech Technical University, Prague, Czech Republic, in 1973. Currently, he is a Professor with the University of West Bohemia, Pilsen, Czech Republic. His research interests are mathematical and computer modelling of electromagnetic fields and coupled problems in power applications. He is the author or coauthor of two monographs (USA), approximately 500 research papers, and several large codes. 